\title{
A reexamination of the effects of motivational state on utilization of conspecific odors in the rat
}

\author{
MARY N. TRAVIS \\ Kansas Wesleyan University, Salina, Kansas \\ H. WAYNE LUDVIGSON \\ Texas Christian University, Fort Worth, Texas \\ and \\ PAUL J. ESLINGER \\ Brown University, Providence, Rhode Island
}

\begin{abstract}
Two previous studies suggested that rats are unable to use conspecifics' odors when the motivational state of the donor rats is different from their own (Davis et al., 1974; Davis, Prytula, Noble, \& Mollenhour, 1976). The effects of motivational conditions on the utilization of such odors were further evaluated in the present study. In Phase 1, test rats were given training either with donors in the same motivational state or with donors in a different state. Differential responses to donors' odor emissions occurred only when the motivational states of donors and test subjects were the same, thus confirming earlier findings. However, when test subjects' motivational conditions were changed in Phase 2, discriminations that had been present in Phase 1 were maintained even though the motivational states of these test subjects and their respective donors no longer matched. Thus, any constraint on the rat's ability to use odors from conspecifics in a different motivational state is not absolute.
\end{abstract}

The fact that rats receiving rewarded and nonrewarded trials in a straight alleyway produce odors that can serve as sources of behavioral control for conspecifics has received substantial support (Ludvigson, 1969; Ludvigson \& Sytsma, 1967; McHose \& Ludvigson, 1966; Seago, Ludvigson, \& Remley, 1970). Odors arising from rewarded treatments have been shown to produce unconditioned approach reactions (Mellgren, Fouts, \& Martin, 1973), whereas odors arising from nonrewarded treatments have been shown to produce unconditioned avoidance reactions (Collerain \& Ludvigson, 1977; Ludvigson, McNeese, \& Collerain, 1979). Furthermore, several studies have demonstrated that rats can successfully use one or both of these odors as cues for differential responding (Bloom \& Phillips, 1973; Ludvigson, 1969; Ludvigson \& Sytsma, 1967; Prytula \& Davis, 1974).

The ability of rats to use odors as discriminative stimuli has raised some interesting questions concerning the possible limits of that control. Davis et al. (1974) and Davis, Prytula, Noble, and Mollenhour (1976) provided evidence that successful use of odors as cues for responding may be related to specific conditions associated with foodversus water-deprivation and/or reward. Using startboxplaced donor subjects, Davis et al. (1974) and Davis et al. (1976) found that runway-trained test subjects responded

Address correspondence to Mary N. Travis, Department of Behavioral Science, Kansas Wesleyan University, Salina, KS 67401. differentially to donors' odors (i.e., they exited the startbox rapidly on rewarded trials and slowly on nonrewarded trials) only when the deprivation states and associated reinforcers of donor-test pairs were the same. More specifically, during Phases 1 and 2 of those initial experiments, donors and test subjects were maintained in different deprivation states. Test subjects failed to respond differentially to donor-generated odors during Phase 1 when the schedule of reward and nonreward events for donors and test subjects was the same or during Phase 2 when donors were rewarded on trials on which test subjects were nonrewarded and vice versa. In Phase 3, the deprivation state of each test subject was shifted to match that of its respective donor subject. After the shift in deprivation state was made, test subjects responded differentially to donors' odors.

These results suggested a constraint on the rat's ability to utilize odors from conspecifics experiencing a different state of deprivation and/or reward. More particularly, such odors appear to be serviceable as cues for differential responding only when the motivational states of donors and test subjects are the same. ${ }^{1}$ Of potential significance, however, is the fact that the studies of Davis et al. (1974) and Davis et al. (1976) did not rule out the possibility that insufficient training might account for the difficulty their test subjects experienced in utilizing odors from donors maintained in a different motivational state. Since no test subjects were given initial training with donors in the same motivational state, the appearance of discriminations in the last phase of these studies might be attributable to the 
sheer number of trials administered, rather than to the motivational conditions of donors and test subjects.

In a recent series of experiments, Weaver and Davis (1983) found that rats may, in fact, be capable of utilizing odors from conspecifics maintained in a different deprivation state. Specifically, Weaver and Davis reported that when both food- and water-deprived subjects were run together in one squad and reinforced with the same substance (a $32 \%$ sucrose-water solution), discriminations developed in the goal measure. However, since the procedures used by Weaver and Davis were quite different from those used earlier by Davis et al. (1974) and Davis et al. (1976) - that is, donors were not used to supply odor cues in the startbox and a single reinforcer was used-it is not clear why test subjects in the earlier studies failed to discriminate donors' odors when the motivational states of donors and test subjects were different.

The present experiment utilized the procedures described by Davis et al. (1974) and Davis et al. (1976) and further evaluated the apparent limits specific motivational conditions impose on the discriminative use of conspecific odors by the rat. The experiment's purpose was threefold. First, it was necessary to determine whether the basic findings reported by Davis et al. (1974) and Davis et al. (1976) could be replicated. Second, the present study explored whether insufficient training could explain the absence of discriminations in the earlier studies when the motivational states of donors and test subjects were different. Third, if rats are indeed impaired in their ability to use odors from conspecifics in a different motivational state, a question might be raised regarding the rat's ability to use such "incongruent" odors if given initial training with those odor stimuli as "congruent." More specifically, it would be of interest to determine whether motivational conditions affect both the acquisition and the maintenance of odor-based discriminations, or only the acquisition of such discriminations.

\section{METHOD}

\section{Subjects}

The subjects were 56 naive female rats obtained from the Sprague Dawley Company, Madison, Wisconsin. All rats were approximately 90 days old at the beginning of their experimental training. All subjects were individually housed and were maintained under conditions of constant illumination.

\section{Apparatus}

The apparatus was a single straight alleyway, which has been fully described elsewhere (Eslinger \& Ludvigson, 1980). A series of photocells located $.15,1.36$, and $1.66 \mathrm{~m}$ beyond the start door allowed for the automatic recording of start, run, and goal traversal times. Stainless steel inserts, designed to fit snugly inside the startbox and goalbox, were used in conjunction with a vacuum cleaner and disposable paper flooring as experimental controls for odor emissions. Stainless steel food cups were used to hold food in the startbox and goalbox. When water was used as a reinforceing agent, opaque glass cylinders were used to contain it.

\section{Procedure}

The experiment was conducted in two segments, each requiring 28 animals. In the first segment of the experiment, 28 subjects were randomly assigned to one of two equal groups of 14 animals each, the odor-donor and test groups. The odor-donor subjects were then randomly assigned to two subgroups of 7 animals each, food deprived and water deprived. The 14 test subjects were also randomly assigned to two groups of 7 subjects each, Groups WaterWater-Water-Food (WW-WF) and Food-Water-Food-Food (FW-FF). One donor from the food-deprived donor group was permanently assigned to each test subject in Group FW-FF. Similarly, one donor from the water-deprived donor group was permanently assigned to each test subject in Group WW-WF. The food and water designations for the two groups of test subjects refer to the deprivation states of the test subjects and their paired donor subjects. For example, each test subject in Group WW-WF was paired with a water-deprived donor subject, as indicated by the first $W$ in the group designation. Each test subject in that group was given initial training while water deprived, as indicated by the second $W$. The $\mathrm{W}$ and $\mathrm{F}$ following the dash signal the deprivation states of donors and test subjects, respectively, following a shift in test subjects' deprivation conditions after Phase 1. Donors for Group WW-WF were maintained on water deprivation throughout the experiment, whereas test subjects were shifted from water deprivation to food deprivation after Phase 1. Donors for Group FW-FF were also maintained in their initial deprivation state throughout, whereas their test-subject counterparts experienced a shift in deprivation state after Phase 1. Consequently, test subjects from Group WW-WF were given initial training with congruent donors and subsequent training with incongruent donors. Conversely, test subjects from Group FW-FF were originally trained with incongruent donors and subsequently trained with congruent donors.

A 7-day pretraining period preceded the beginning of Phase 1 training. During that period, each subject was either placed on a 23-h water deprivation schedule or reduced to approximately $85 \%$ of its free-feeding body weight. All animals were handled and tamed during those 7 days. On the last 2 pretraining days, all animals from the two test-subject groups were given 5 min of runway exploration, with all equipment operating and the goalbox unbaited. Each test subject was also allowed a 5-min habituation period in the home cage to an opaque glass cylinder filled with water on the last 2 days of pretraining. All animals from the two odor-donor groups were given eight direct startbox placements on the last 2 pretraining days, according to a double alternation schedule of reward (R) and nonreward $(N)$, that is, RRNNRRNN. Water-deprived odor donors were given $30 \mathrm{sec}$ access to a full cylinder of water on $R$ trials and $30 \mathrm{sec}$ confinement to an empty startbox on $\mathrm{N}$ trials. Similarly, fooddeprived odor donors were given $30 \mathrm{sec}$ access to a trough filled with 45-mg Noyes pellets on $R$ trials and were confined to an empty startbox for $30 \mathrm{sec}$ on $\mathrm{N}$ trials.

During Phase 1 ( 18 days), all subjects were given trials at the rate of eight per day. Reward and nonreward events followed a RRNNRRNN schedule for 2 days followed by a NNRRNNRR schedule for 2 days. The reward/nonreward schedule was the same for all animals on any given day. Test animals from Group WW-WF and their donors always received their daily trials before test animals from Group FW-FF and their donors received any trials. This procedure was followed to ensure that animals on water deprivation had been deprived for $23 \mathrm{~h}$ at the beginning of each experimental session. The order of running donor-test pairs within each group was randomized daily, but remained constant within a day.

A trial during Phase 1 began with placement of the appropriate odor donor into the startbox. On R trials, donors were given $30 \mathrm{sec}$ access to a stainless steel trough filled with $45-\mathrm{mg}$ Noyes pellets or to a full cylinder of water. On $\mathrm{N}$ trials, donors were confined to an empty startbox for $30 \mathrm{sec}$. Immediately upon removal of the donor, the appropriate test subject was placed into the startbox. After $5 \mathrm{sec}$, the start gate was raised, allowing the test subject to traverse the alleyway. On R trials, test subjects were given $30 \mathrm{sec}$ access to water, and on $\mathrm{N}$ trials, they were confined to the goalbox with an empty water container for $30 \mathrm{sec}$. After each donor-test pair 
was run, a clean stainless steel insert was positioned inside the startbox, and the paper flooring inside the startbox was replaced. After all donor-test pairs in a group completed a trial, the entire runway was cleaned: the air was exhausted for $60 \mathrm{sec}$, stainless steel inserts in both goal and start sections were replaced by clean ones, and the paper flooring throughout the entire runway was replaced.

Upon completion of Phase 1 , all subjects were given 1 day of free access to food and water. A 5-day shift period followed during which each test subject was reduced to approximately $85 \%$ of its free-feeding body weight. Test subjects were allowed free access to water for the remainder of the experiment. Donors were maintained on the same deprivation schedule as in Phase 1. Test subjects were habituated to a stainless steel trough filled with 45 mg Noyes pellets for $5 \mathrm{~min}$ in their home cages on the last 2 shift days.

Phase 2 ( 12 days) immediately followed the 5-day shift period. Procedures for a trial were identical to those described for Phase 1 , with one exception. On R trials, 15 45-mg Noyes pellets were dispensed to test subjects as soon as the last photobeam was broken. The subjects were allowed $30 \mathrm{sec}$ to consume the reward. On $\mathrm{N}$ trials, the subjects were confined to the goalbox with an empty food trough for $30 \mathrm{sec}$.

The second segment of the experiment was conducted similarly to the first segment. The second segment not only provided a replication of the first, but also served to counterbalance the experimental design. As was the case in the first segment, 28 subjects were randomly assigned to two equal groups, odor-donor and test. The odordonor subjects were divided as before into two equal subgroups, water deprived and food deprived. The test subjects were also divided into two equal groups, Groups Food-Food-Food-Water (FF-FW) and Water-Food-Water-Water (WF-WW). The group designations for test subjects were as described above.

All experimental procedures were the same as previously described, with three exceptions. First, test animals in Group FF-FW and their donors completed all training before test animals in Group WF-WW and their donors began training. This change in procedure was necessary due to time constraints. Second, during the 5-day shift phase, each test animal was given food ad lib and was placed on a $23-\mathrm{h}$ water deprivation schedule. In the first segment of the experiment, test animals were shifted from water to food deprivation. Third, test subjects were habituated to food in their home cages during pretraining and to water in their home cages during the deprivation-shift period. (In the first segment, test subjects had been habituated to water during pretraining and to food during the deprivation-shift period.) Phase 1 lasted 18 days, and Phase 2 continued for 12 days, as was the case in the first segment of the experiment.

\section{RESULTS}

Three runway traversal latencies (start, run, and goal) were recorded for each test subject for each trial during both Phases 1 and 2 . These latencies were converted to reciprocals and multiplied by the appropriate constants to yield speed scores in meters per second. Mean R and $\mathrm{N}$ speeds were then calculated for each test-subject group for each training day. These means reflect a daily average of the 7 test animals' speeds within each of the four groups for the four $\mathbf{R}$ and four $\mathbf{N}$ trials.

Analyses of variance were performed on start speeds for both phases of the experiment. These analyses included as factors cue (food-deprived donor vs. water-deprived donor), deprivation state of test subject (food vs. water deprived), goal events (R vs. $\mathbf{N}$ ), and days.

Figure 1 shows the mean start speeds for the four testsubject groups in Phases 1 and 2. Analysis of start speeds for Phase 1 included Days 13-18. By that time, perfor-

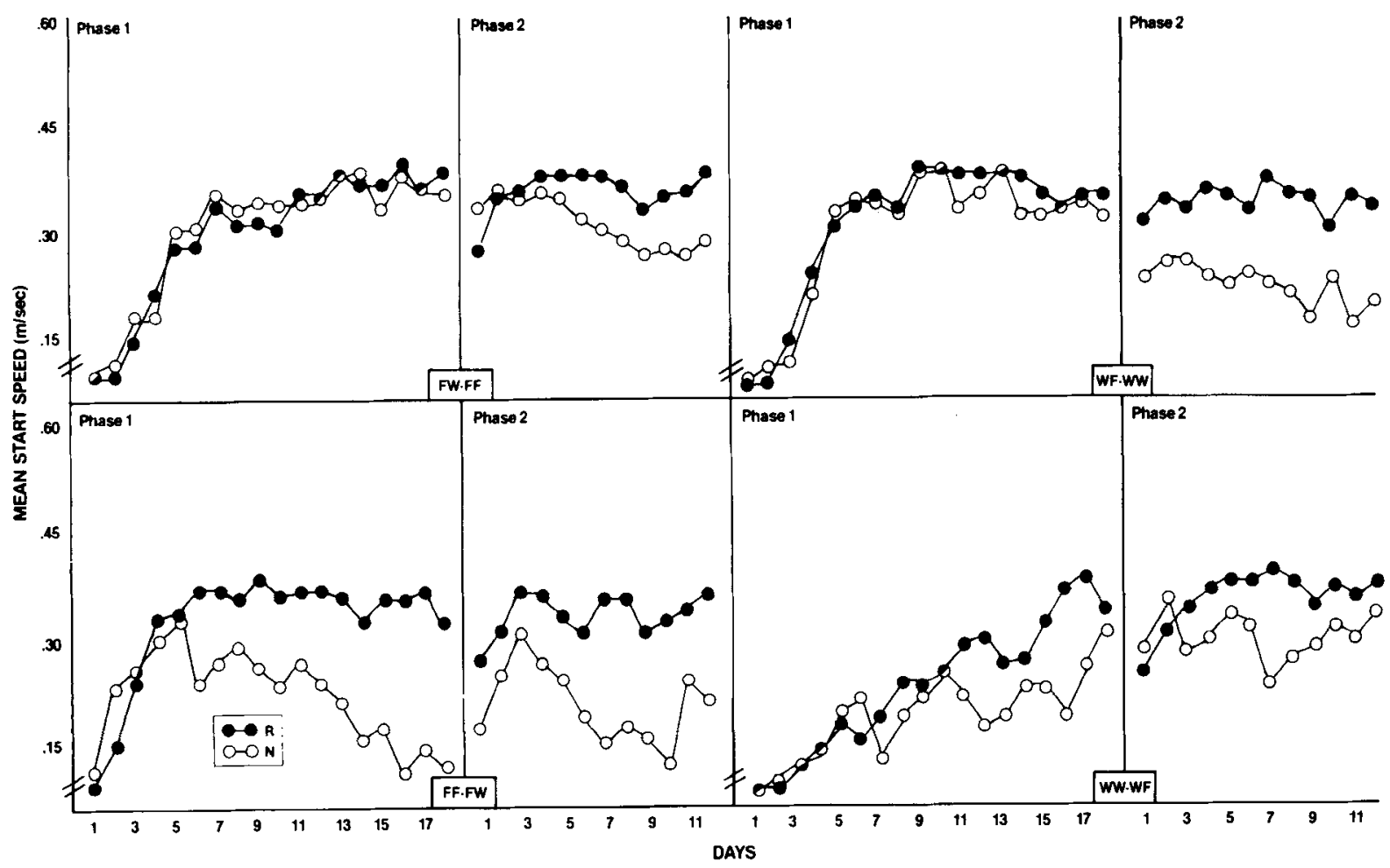

Figure 1. Mean start speeds (in meters per second) for Phases 1 and 2 for Groups FF-FW, FW-FF, WW-WF, and WF-WW. 
mance of the four groups was stable in that alleyway measure. This analysis revealed that the four groups differed in overall presence or absence of significant $R$ versus $\mathbf{N}$ discriminations $[F(1,24)=73.52, p<.001]$. Groups FF-FW and WW-WF had significantly faster speeds on rewarded than on nonrewarded trials $\left[F_{\mathrm{s}}(1,24)=186.06\right.$ and 36.52 , respectively; $p s<.001$ ]. Groups FW-FF and WF-WW, on the other hand, failed to show significant differences between $\mathrm{R}$ and $\mathrm{N}$ speeds in the start measure on those days. Further analysis revealed that $\mathbf{N}$ speeds varied significantly among the groups $[F(1,48)=71.80$, $p<.001]$, whereas $\mathrm{R}$ speeds were not significantly different. Differences in $\mathbf{N}$ speeds were further probed with the Newman-Keuls procedure. Group FF-FW had significantly slower speeds than all other groups on nonrewarded trials $(p<.01)$. Similarly, Group WW-WF ran significantly more slowly on $\mathrm{N}$ trials than either Group FW-FF or Group WF-WW $(p<.01)$.

Differences in start speeds for $\mathbf{R}$ and $\mathrm{N}$ trials during Phase 1 were also found to vary for the four groups in relation to day of training $[F(5,120)=3.33, p<.01]$. Group FF-FW ran significantly faster on rewarded than on nonrewarded trials on Days 13-18 $\left[F_{\mathrm{s}}(1,144)=29.53\right.$, $38.64,46.31,86.45,70.46$, and 58.92 , respectively; $p s<.001]$. Group WW-WF showed significant $\mathrm{R}$ versus $\mathrm{N}$ differences on Days $13,15,16$, and $17[F \mathrm{~s}(1,144)=$ $6.32,11.12,41.88$, and 19.81 , respectively; $p s<.025$, $.001,<.001$, and $<.001$, respectively]. Groups FF-FW and WF-WW failed to show discriminations on any of the last 6 days of Phase 1 .

Analysis of Phase 2 start speeds included all 12 days of training, because it was important to assess the effects of shifting test subjects' deprivation states on discriminations in the start measure from the outset of that training. This analysis revealed that the discriminations of foodand water-deprived test subjects differed in magnitude $[F(1,24)=19.55, p<.001]$. Although both fooddeprived subjects (Groups FW-FF and WW-WF) and water-deprived subjects (Groups WF-WW and FF-FW) showed significant differences between speeds on $R$ and $N$ trials during Phase $2[F \mathrm{~s}(1,24)=16.83$ and 108.76 , respectively; ps $<.001$ ], water-deprived subjects ran significantly more slowly than food-deprived subjects on $\mathbf{N}$ trials $[F(1,48)=22.99, p<.001]$. Their speeds on $R$ trials, on the other hand, were not significantly different.

Although all groups clearly showed an overall discrimination between rewarded and nonrewarded treatments in the start measure during Phase 2, the analysis involving all 12 days of Phase 2 failed to pick up the general absence of discriminations for Groups FW-FF and WW-WF on the first 2 days of Phase 2. It may be worth noting that both of these groups failed to display significant $R$ versus $\mathbf{N}$ differences on the last day of Phase 1 . It is also interesting that Group WF-WW displayed faster speeds on $\mathbf{R}$ trials than on $\mathbf{N}$ trials on the first 2 days of Phase 2, despite the fact that no evidence of discriminations was detected during Phase 1 . Therefore, a separate analysis of variance that included only the first 2 days of
Phase 2 was performed. This analysis revealed that there were differences in the $\mathbf{R}$ and $\mathrm{N}$ speeds of food- and waterdeprived test subjects on those days $\left[F_{\mathrm{s}}(1,24)=15.54\right.$, $p<.001$ ]. Water-deprived subjects (Groups WF-WW and $\mathrm{FF}-\mathrm{FW}$ ) showed significant $\mathrm{R}$ versus $\mathrm{N}$ differences on those days $[F(1,24)=14.79, p<.001]$, whereas food-deprived subjects (Groups FW-FF and WW-WF) failed to respond differentially to $R$ and $N$ treatments. Further probing revealed that $\mathrm{N}$ speeds were significantly slower for water-deprived than for food-deprived subjects $[F(1,24)=15.29, p<.001]$. Their speeds on $\mathbf{R}$ trials did not differ.

Figure 2 shows the mean goal speeds for the four testsubject groups in Phases 1 and 2. Analyses of variance like those performed on start speeds were also used to examine goal speeds; however, because these data were not central to the hypotheses under test, only the major results are considered here. Analysis of goal speeds for the last 6 days of Phase 1 revealed significant discriminations for Groups FF-FW, FW-FF, WW-WF, and WF-WW $\left[F_{\mathrm{S}}(1,24)=46.59,13.70,6.85\right.$, and 91.43 , respectively; $p$ s $<.001,<.001,<.025$, and $<.001$, respectively]. Speeds on $N$ trials were slower for fooddeprived test subjects (Groups FF-FW and WF-WW) than for water-deprived test subjects (Groups WW-WF and FW-FF) $[F(1,48)=14.25, p<.001]$.

Analysis of goal speeds for the 12 days of Phase 2 also confirmed the presence of significant discriminations between rewarded and nonrewarded trials for all four groups $[F \mathrm{~s}(1,24)=40.00,86.12,12.84$, and 174.84 for Groups FF-FW, FW-FF, WW-WF, and WF-WW, respectively; ps $<.001,<.001,<.01$, and $<.001$, respectively].

\section{DISCUSSION}

The present experiment addressed three primary questions: (1) Could the basic findings of Davis et al. (1974) and Davis et al. (1976) be replicated? (2) Could the apparent difficulty that test subjects experienced in discriminating odors provided by donors in a different deprivation state and rewarded with a different agent be an artifact of insufficient training in those studies? (3) If rats are truly limited in their ability to utilize such incongruent odors, would the maintenance of previously established discriminations be affected, or would the acquisition of initial discriminations alone be impaired? An inspection of the present data and results affords insight into these three issues.

The start data from Phase 1 clearly show that test subjects paired with donors experiencing different conditions of deprivation and reward (Groups FW-FF and WF-WW) failed to show reliable evidence of differential responding by the end of the 18-day acquisition period. Only in Phase 2, when motivational states of donors and test subjects matched, did discriminations based on donorgenerated odors emerge in those two groups. On the other hand, test subjects paired with congruent donors (Groups FF-FW and WW-WF) displayed significant discrimina- 


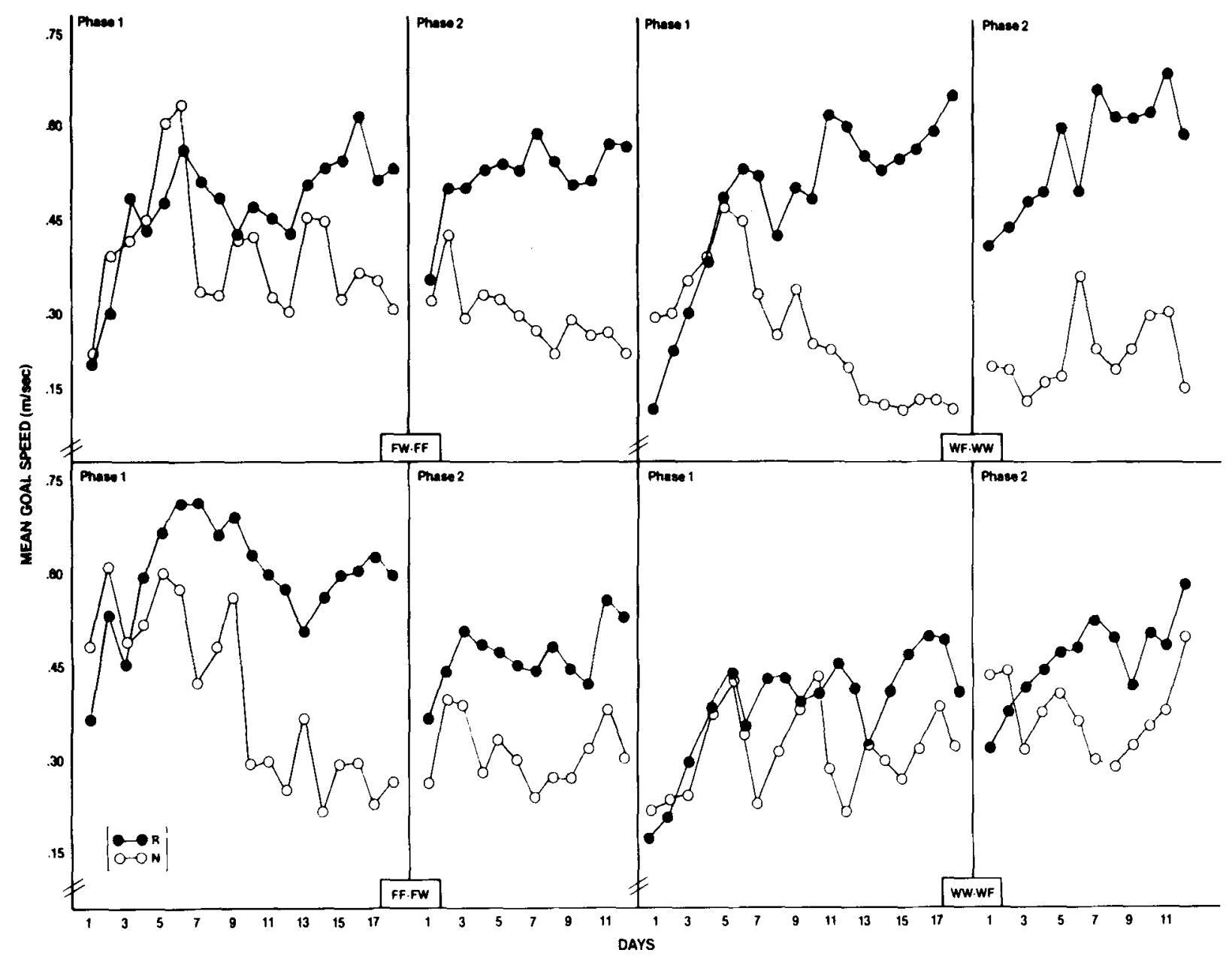

Figure 2. Mean goal speeds (in meters per second) for Phases 1 and 2 for Groups FF-FW, FW-FF, WW-WF, and WF-WW.

tions between rewarded and nonrewarded treatments on several of those days. Group FF-FW, in particular, showed large differences between $\mathrm{R}$ and $\mathrm{N}$ speeds for Days 13-18, the days included in the formal statistical analyses, as well as for several days prior to Day 13. The present data, then, support the basic findings of Davis et al. (1974) and Davis et al. (1976) that odors arising from rewarded and/or nonrewarded treatments of rats in a straight alleyway appear to be motivationally specific. This conclusion derives additional support from the fact that analysis of goal speeds for the last 6 days revealed that all four groups of test subjects showed significant discriminations between rewarded and nonrewarded events in this measure. These discriminations were presumably based on congruent odors produced by the test subjects themselves.

The present results also indicate that the inability of the test rats in the studies of Davis et al. (1974) and Davis et al. (1976) to utilize incongruent donors' odors was not simply due to insufficient training. The fact that Groups FF-FW and WW-WF displayed significant discriminations in the start measure during Phase 1, while Groups FW-FF and WF-WW failed to show reliable differences in speeds on $\mathrm{R}$ and $\mathrm{N}$ trials, demonstrates that length of training alone did not account for the lack of discriminations in the studies of Davis et al. (1974) and Davis et al. (1976).

With regard to the question of whether incongruent motivational conditions would interfere with the maintenance of discriminations established when donors and test subjects experienced congruent conditions, the results of Phase 2 analyses of start speeds revealed that both testsubject groups shifted to motivational conditions incongruent with their donors' (Groups FF-FW and WW-WF) maintained their discriminations during Phase 2. Only on the first 2 days of Phase 2 were discriminations completely abolished, and then only for Group WW-WF. Group FF-FW had faster $R$ speeds than $N$ speeds on each day of Phase 2. Clearly, motivational conditions do not impose an absolute limit on the discriminative use of odor cues.

Although not one of the major concerns of the present study, differences between the responses of food- and water-deprived test subjects to conspecifics' odors were noteworthy. When congruent odors were available and discriminations were present during initial Phase 1 training, food-deprived subjects displayed larger discrimina- 
tions than did their water-deprived counterparts. This bias occurred in both start and goal measures. During Phase 2, the converse occurred-water-deprived test subjects displayed larger discriminations than food-deprived subjects in the start and goal measures. Weaver and Davis (1983) found that water-deprived rats established odor-based discriminations more slowly than did food-deprived rats. They suggested that odors associated with water deprivation and reward may be "less salient and/or intense" than food-related odors. If, in fact, food- and water-related odors do vary in their ability to serve as cues for differential responding, test subjects receiving initial training while food deprived (all food-deprived subjects were exposed to food-related odors in the goalbox) may have experienced an initial learning advantage that carried forward into Phase 2 . The fact that training on a relatively simple problem facilitates the subsequent learning of a more difficult one has been well documented (Haberlandt, 1971; Lawrence, 1952; Sutherland, Mackintosh, \& Mackintosh, 1963).

Although the present experiment provides answers to several questions relating to the rat's ability to utilize conspecific odors as cues for differential responding when motivational states are varied, a number of perplexing questions remain unanswered. First, although the present data demonstrate that some constraint exists on the rat's ability to utilize motivationally incongruent odors, they do not permit an assessment of why such a constraint exists. Second, they do not reveal whether motivational conditions affect odors associated with rewarded treatments, nonrewarded treatments, or both. Third, the present data also do not permit a determination of whether odors varied because of deprivation conditions per se or because of the specific rewarding agents used.

Clearly, additional research is needed to provide answers to these specific questions concerning motivational states and utilization of conspecific odors in rats. More generally, a greater understanding of the olfactory communication network in rats is needed. A study conducted by Galef, Kennett, and Wigmore (1984) revealed that both domestic rats and first-generation laboratory-bred wild rats could transmit to conspecifics information about unique diets they had eaten. If the mechanism of that communication is olfactory, such results would indicate that rats have an elaborate system of odor-based communication. Additional research is needed to clarify the nature and extent of such communication in rats and to describe the relationship of such variables as motivational state to this communication system.

\section{REFERENCES}

BLOом, J. M., \&HILlPS, J. M. (1973). Conspecific odors as discriminative stimuli in the rat. Behavioral Biology, 8, 279-283.

Collerain, I., Ludvigson, H. W. (1977). Hurdle-jump responding in the rat as a function of conspecific odor of reward and nonreward. Animal Learning \& Behavior, 5, 177-183.

Dayis, S. F., Prytula, R. E., Harper, W. E., Tucker, H. K. LEWIS, C., FLOOD, L. (1974). Double-alternation runway performance as a function of inter- and intra-reinforcement odor cues. Psychological Reports, 35, 787-793.

Davis, S. F., Prytula, R. E., Nogle, J. J., Mollenhour, M. N. (1976). Motivational specificity of the signal value of odor cues. Animal Learning \& Behavior, 4, 407-410.

Eslinger, P. J., Ludvigson, H. W. (1980). Are there constraints on learned responses to odors from rewarded and nonrewarded rats? Animal Learning \& Behavior, 8, 452-456.

Galef, B. G., KennetT, D. J., \&igmore, S. W. (1984). Transfer of information concerning distant food in rats: A robust phenomenon. Animal Learning \& Behavior, 12, 292-296.

HaberLANDT, K. (1971). Transfer along a continuum in classical conditioning. Learning \& Motivation, 2, 164-172.

LAWRENCE, D. H. (1952). The transfer of a discrimination along a continuum. Journal of Comparative \& Physiological Psychology, 45, $511-516$

LudvigSon, H. W. (1969). Runway behavior of the rat as a function of inter-subject reward contingencies and constancy of daily reward schedule. Psychonomic Science, 15, 41-43.

Ludvigson, H. W., MCNeese, R. R., \& Collerain, I. (1979). Longterm reaction of the rat to conspecific (frustration) odor. Animal Learming \& Behavior, 7, 251-258.

Ludvigson, H. W., \&YTSMA, D. (1967). The sweet smell of success: Apparent double alternation in the rat. Psychonomic Science, 9. 283-284.

McHose, J. H., \& Ludvigson, H. W. (1966). Differential conditioning with nondifferential reinforcement. Psychonomic Science, 6, 485-486.

Mellgren, R. L., Fouts, R. S., \& Martin, J. W. (1973). Approach and escape to conspecific odors of reward and nonreward in rats. Animal Learning \& Behavior, 1, 129-132.

Prytula, R. E., Davis, S. F. (1974). Runway performance as a function of positively and negatively correlated olfactory cues. Psychological Reports, 35, 735-740.

Seago, J. D., Ludvigson, H. W., Remly, N. R. (1970). Effects of anosmia on apparent double alternation in the rat. Journal of Comparative \& Physiological Psychology, 71, 435-442.

Sutherland, N. S., Mackintosh, N. J., Mackintosh, J. (1963). Simultaneous discrimination training of Octopus and transfer of discrimination along a continuum. Journal of Comparative \& Physiological Psychology, 61, 198-207.

WeAver, M. S., DAvis, S. F. (1983). Motivational specificity of the signal value of odor cues: A reconsideration and extension. Emporia State Research Studies, 32, 5-48.

\section{NOTE}

1. For convenience, motivational state or motivational condition will refer both to a specific type of deprivation and to a particular reinforcing agent. 